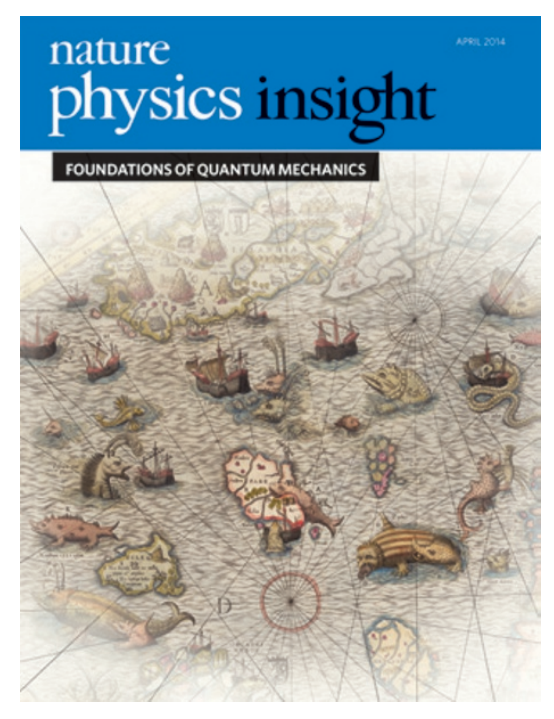

\section{COVER IMAGE}

From science to technology and back again: state-of-the-art tools developed for quantum technologies, in theory and experiment, are allowing researchers to revisit the foundations of quantum theory and to explore the terra incognita that may lie beyond.

IMAGE: CARTA MARINA, OPUS OLAI MAGNI GOTTI LINCOPENSIS, EX TYPIS ANTONII LAFRERI SEQUANI, ROM, 1572 COLOURED ENGRAVING, NATIONAL LIBRARY OF SWEDEN, MAP COLLECTION, KOB, KARTOR, 1 AB

\section{NPG LONDON}

The Macmillan Building,

4 Crinan Street, London N1 9XW

$\mathrm{T}:+442078334000$

F: +442078434563

naturephysics@nature.com

EDITOR

ALISON WRIGHT

INSIGHT EDITOR

IULIA GEORGESCU

PRODUCTION/ART EDITOR

ALLEN BEATTIE

COPY EDITOR

KEVIN SHERIDAN

SENIOR COPY EDITOR

JANE MORRIS

EDITORIAL ASSISTANT

EMMA BURCH

MARKETING

SARAH-JANE BALDOCK

PUBLISHER

RUTH WILSON

EDITOR-IN-CHIEF,

NATURE PUBLICATIONS

PHILIP CAMPBELL

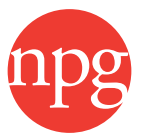

nature publishing group

\title{
Foundations of quantum mechanics
}

T he fields of quantum information theory and quantum technology exploded in the late 1990s the very decade that marked the rise of the internet. Labelled the 'second quantum revolution', this new wave of multidisciplinary research was fuelled by the quest for faster computers and secure communication. But exploiting purely quantum mechanical features for information processing requires a deeper understanding of their origin and role in different physical systems, as well as exquisite experimental control.

More than two decades of research have resulted in remarkable theoretical progress and experimental capabilities that now enable us to revisit the very foundations of quantum theory. To make a cartographic analogy, our present understanding of quantum mechanics is like an island containing still uncharted regions and with indistinct coastlines; even less is known of what may lie beyond the surrounding seas. This Nature Physics Insight covers some of the exploratory attempts to improve our map of the quantum world.

Experimental advances in the creation of macroscopic superposition states are pushing the limits of quantum theory to establish whether (or where) the quantum description eventually breaks down and the classical one takes over. Such studies might even betray gravitational corrections to quantum mechanics and could therefore be useful in quantum gravity research. In parallel, photonic experiments are providing new insight into nonlocality and complementarity - recent work seems to suggest that these too could be exploited to test models of quantum gravity, taking that quest from astrophysical observations to Earth-based experiments.

On the theoretical side, intriguing concepts are emerging - such as possible nonlocal correlations that are stronger than those predicted by quantum mechanics, or the existence of an indefinite causal structure. These concepts could be exploited in new quantum information processing tasks, and they illustrate the two-way relationship that exists between quantum information theory and the foundations of quantum mechanics. And, as we celebrate fifty years of Bell's theorem this year, it seems timely to consider entanglement and its previously unsuspected connections to other areas of physics, such as thermodynamics and many-body theory.

It would be impossible to cover all of the exciting research directions in this very active field, hence the aim of this Insight on the foundations of quantum mechanics is to provide merely a taste and to encourage a deeper exploration of the subject.

Iulia Georgescu, Associate Editor

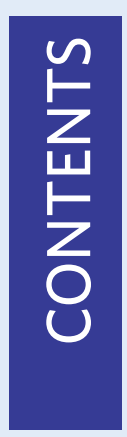

\section{COMMENTARY}

Gravity in quantum mechanics

Giovanni Amelino-Camelia

Quantum entanglement

Vlatko Vedral

PROGRESS ARTICLE

Quantum causality

Časlav Brukner

\section{REVIEW ARTICLES}

Nonlocality beyond quantum mechanics

Sandu Popescu

Testing the limits of quantum mechanical superpositions

Markus Arndt and Klaus Hornberger

Testing foundations of quantum mechanics with photons

Peter Shadbolt, Jonathan C. F. Mathews, Anthony Laing and Jeremy L. O'Brien 